\title{
What is the True Location of Jacoby's Line?
}

\author{
By \\ Naoki SHIRAISHI and George MATSUMURA

\begin{abstract}
Kyorin University, School of Medicine, Department of Anatomy, Shinkawa 6-20-2, Mitaka, Tokyo 181-8611, Japan e-mail: nsmd@kyorin-u.ac.jp
\end{abstract} \\ - Received for Publication, August 22, $2005-$ \\ Key Words: Jacoby's line, Intercrestal line, Cadaver study, Conus medullaris, Dura mater

\begin{abstract}
Summary: We investigated 60 cadavers to examine the accurate location of conus medullaris, lower end of dura mater, and intercrestal line (Jacoby's line).

Jacoby's line was established using the method described by Jacoby GW (1899). Then the intersection of this line and vertical line through the spinous processes was marked by a fine nail. After a laminectomy was performed to expose the spinal cord, spinal level of the conus medullaris, the lower end of dura mater, and Jacoby's line were examined, and marked and recorded these locations of nailing.

In 2 of 48 cases $(2.1 \%)$, Jacoby's line passed through the level superior to $3^{\text {rd }}$ lumber vertebra (L3). The Conus medullaris was at the level beneath L3 in 2 of 57 cases (3.5\%). And lower end of dura mater was located beneath the superior margin of $S 3$ in 1 of 28 cases (3.6\%). When Jacoby's line is used as a guide-point, the site for subarachnoid puncture is established in the L3-4 interspinous space. But when we consider the risk of spinal cord injury cannot be completely excluded, in case that conus medullaris is located inferior to $\mathrm{L3}$ in $3.5 \%$ of cases. Conseqently, it should be important to avoid performing subarachnoid puncture at L2-3 or higher lumbar vertebral level especially in the elderly.
\end{abstract}

The eponym 'Jacoby's line' comes from an U.S. physician, George W. Jacoby (1856-1940), who first claimed the clinical significance of the intercrestal line in the New York Medical Journal in 1899 ${ }^{1,2)}$. Its first appearance in the medical literature in Japan was in a 1907 reported by Kida, in which Jacoby's line was used as a guide-point for a trial of spinal anesthesia using cocaine ${ }^{3)}$. Earlier references to Jacoby's line have not been confirmed ${ }^{4}$. Jacoby's original article notes that the line between the top of the bilateral iliac crests passes the level of L4 vertebral body ${ }^{1,2)}$. He also revealed the intersection of this line and midline is an usuful guide-point for subarachnoid puncture. However, descriptions about the vertebral level of Jacoby's line are not consistent in textbooks and/or practical manuals of anesthesiology, neurosurgery, neurology.

In a most frequently used reference book of anesthesiology in Japan 'Masuigkagaku Zensho', Jacoby's line is defined as an imaginary line passing the spinous process of L4 or the space between the L4 and L5 spinous processes ${ }^{5}$. Similarly, Yokoyama (2000) states that "Jacoby's line transverses the spinous process of L4" "6), while Moriyasu notes that "it crosses between L4 and L5"7). In brief, any clear standard about the vertebral level of Jacoby's line has not been established. In addition, anatomical observations on Jacoby's line are remarkably scant except for a few radiological researches. Nor any cadaver studies have been performed in Japanese subjects ${ }^{8,9}$. The aim of the present study is to clarify the position of Jacoby's line using 60 Japanese cadavers, and to confirm the significance of this line as a guide-line for lumber puncture.

\section{Subjects and Methods}

60 Japanese cadaver specimens with no severe compression fractures, scoliosis or other pronounced spinal deformities were examined, ranging from 58 to 96 years of age. The subjects were fixed by infusion of a mixture of formalin, ethanol, and glycerin. On the investigation, the cadavers were put on the prone position. At First, Jacoby's line was established according to his original description (1899), then the intersection of Jacoby's line and the spinal line through the spinous processes was pointed and marked by using a fine nail. Laminectomy was performed carefully to removed the vertebral arches in one piece. After removal of the 


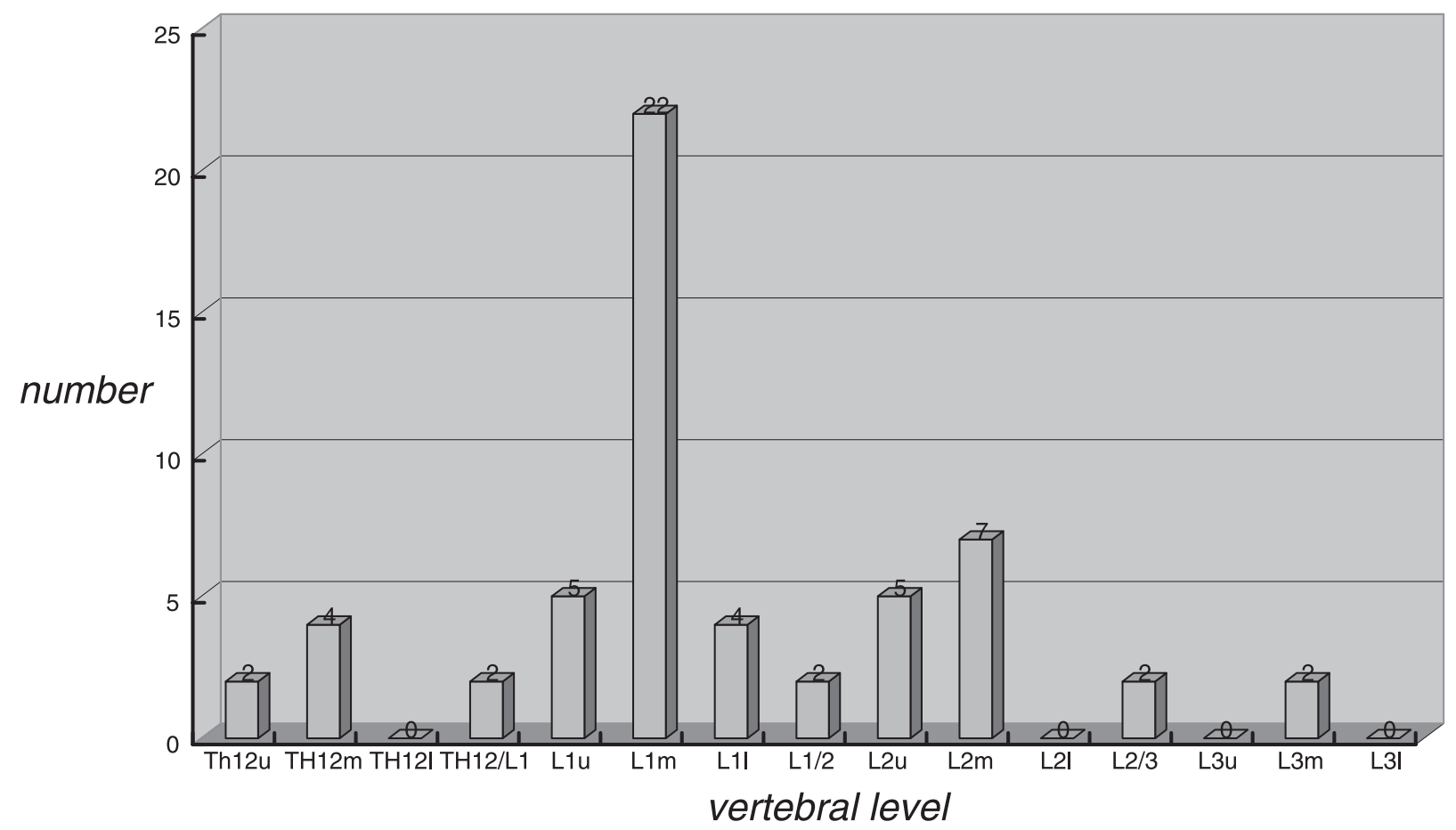

Fig. 1. Level of Conus medullaris.

vertebral arches, dorsal portion of spinal dura was partially incised to expose the spinal cord. While the spinal cord was observed macroscopically, the spinal level of conus medullaris, lower end of dura mater, and the level of Jacoby's line were observed and these locations are made using fine nails on the spine. After that the sites pointed by fine nails were recorded and photographed.

\section{Results}

\section{Conus medullaris}

The position of conus medullaris could be ascertained in 57 of 60 subjects. In 3 subjects conus portion could not be clearly confirmed due to the deformity of the vertebral column and/or poor transfusion of fixative into the spinal cord. The results for the remaining 57 subjects are shown in Fig. 1. Conus medullaris was located between the superior margin of L1 to the inferior margin of L2 in 45 subjects $(78.9 \%)$. Conus medullaris located at L3 or lower were observed in 2 cases $(3.5 \%)$ of 57 subjects.

\section{Lower end of dura mater}

The lower end of dura mater could be confirmed in $28(46.7 \%)$ of 60 subjects, though the ones could not be clearly observed in the remaining 32 sub- jects, because dura mater was destroyed when the dorsal part of sacrum was removed. In $25(89.3 \%)$ of the 28 included subjects, lower end of dura mater was observed at S1 or S2 vertebral level. The lower end of dura mater apparently below the superior border of S3 was found in only 1 case $(3.6 \%)$ of these 28 subjects (Fig. 2).

\section{Jacoby's line}

Jacoby's line was determined in 48 of 60 subjects. Another 12 subjects were excluded since their iliac crests could not be clearly confirmed from body surface due to the severe decubitus ulcers and/or pelvic deformation. Out of these 48 subjects, Jacoby's line was ascertained to pass through the level between superior brim of L4 and inferior brim of L5 in 36 subjects $(75.0 \%)$. Only one case $(2.1 \%)$ with Jacoby's line being located cranial to the inferior brim of the L3 vertebral body was observed (Fig. 3).

\section{Discussion}

The intercrestal line is a guide-line for lumbar puncture first proposed by an US physician G.W. Jacoby ${ }^{1,2)}$, but the eponymous term "Jacoby's line" is used only in Japan and is not found in any reference books or medical literature in the West. Even 


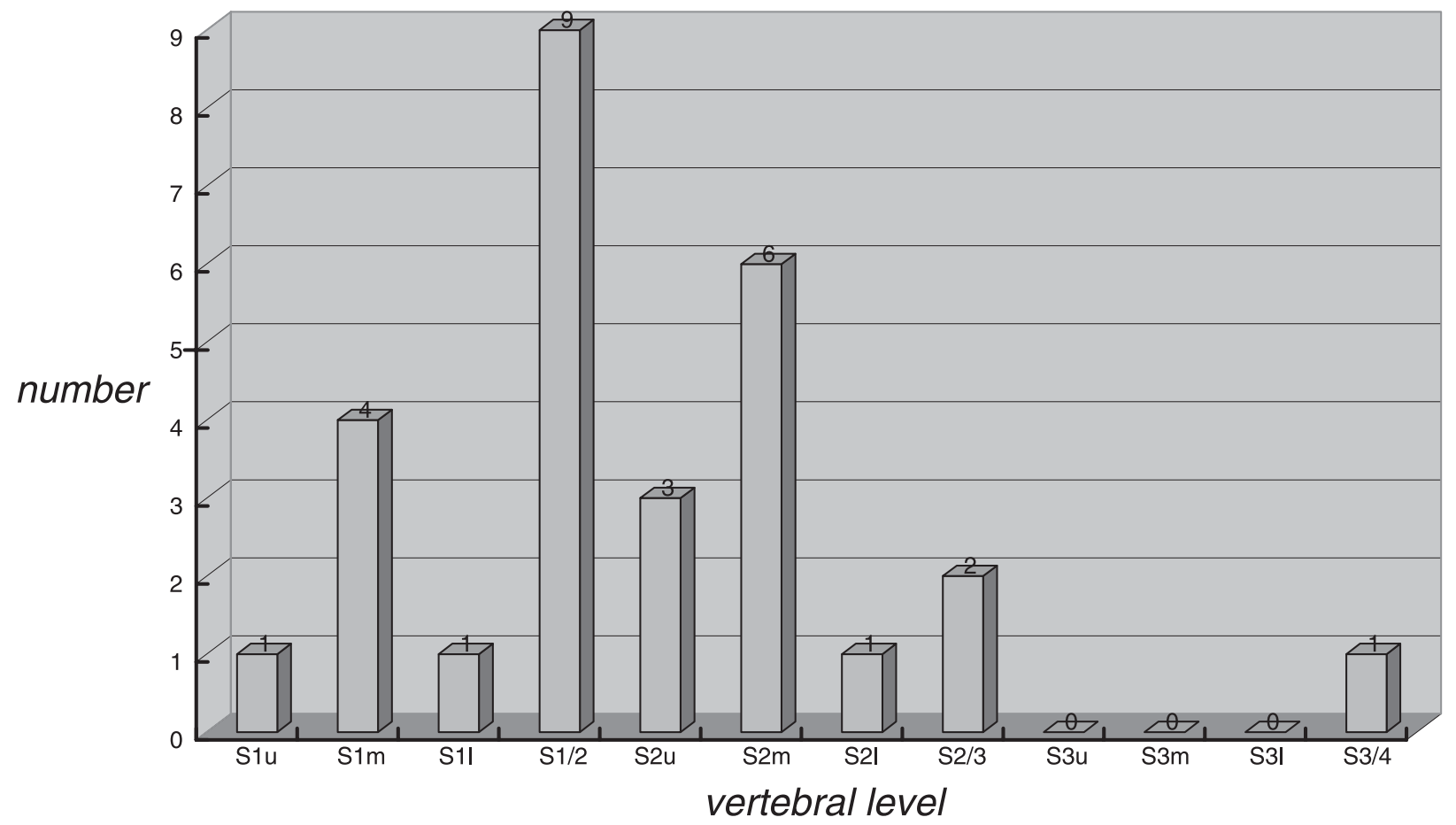

Fig. 2. Level of distal end of Dura mater.

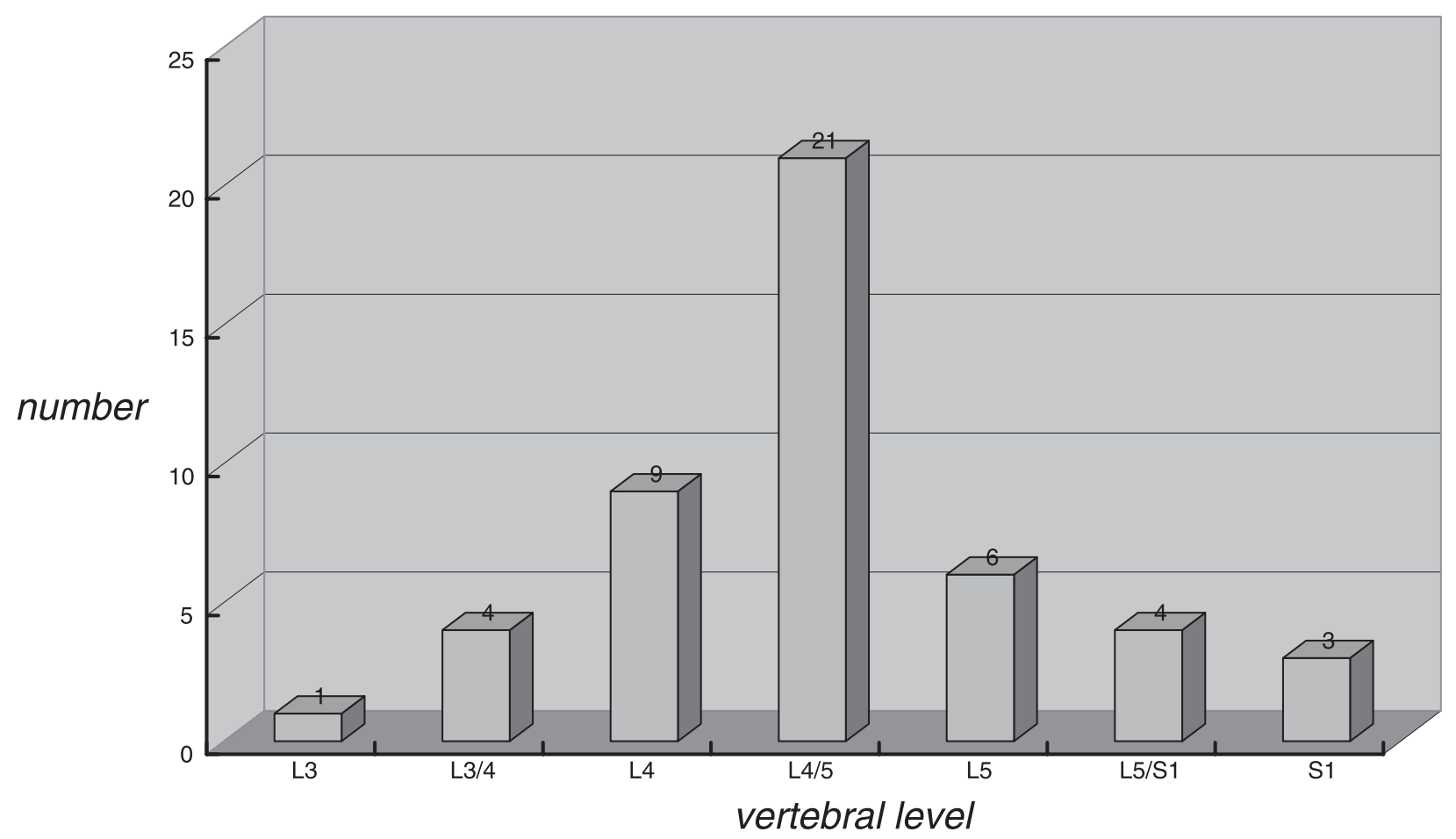

Fig. 3. Level of Jacoby's line. 
Table 1. Comparion of Jacoby's line between Japanese and British

\begin{tabular}{lcc}
\hline & $\begin{array}{c}\text { Japanese } \\
(\mathrm{n}=100)\end{array}$ & $\begin{array}{c}\text { British } \\
(\mathrm{n}=163)\end{array}$ \\
\hline $\begin{array}{l}\text { Location of spine } \\
\text { L3/4 }\end{array}$ & $\mathrm{n}(\%)$ & $\mathrm{n}(\%)$ \\
4th vertebral body & $43(43)$ & $6(3.7)$ \\
L4/5 & $26(26)$ & $79(48.5)$ \\
5th vertebral body & $24(24)$ & $49(30.1)$ \\
L5/S1 & $7(7)$ & $23(14.1)$ \\
\hline
\end{tabular}

in Japan, the term 'Jacoby's line' does not appear in any publications earlier than Kida's study $(1907)^{3)}$, and the first author of the eponym has not been known even now. Additionally, very little information about the position of Jacoby's line has been reported especially in the cadaver studies. In a radiological study on 100 Japanese, Matsuo et al. showed Jacoby's line passing the L4 spinous process level in $43 \%$ of cases, the L4-5 interspinous space in $26 \%$, and the L5 level in $24 \%$ respectively $^{8)}$. Using radiographs of 163 British subjects, Render reported Jacoby's line located at the level of L3-4 interspinous space were observed in 3.7\% of the total (Table 1) ${ }^{9}$. The technique for subarachnoid puncture usually entails palpation of the spinous process of L4 to confirm Jacoby's line ${ }^{5-7,11-12)}$, and the puncture will be performed usually in the intervertebral space between L2 and L3 or between L3 and L4. This method normally results in entry of the needle below conus medullaris, the region in which the cauda equina and the filum terminale are located ${ }^{5-7,11-12)}$.

In the present study, we dissected 60 cadavers to settle the levels of conus medullaris, lower end of dura mater, and Jacoby's line, consequently showed Jacoby's line superior to L3 happened $2.1 \%$ in incidence. These results indicate comprehensively that it may be possible to use Jacoby's line as a guideline for establishing the site of subarachnoid puncture superior to the L3-4 interspinous space. However, in $3.5 \%$ of cases, conus medullaris located beneath the inferior brim of L3 vertebral body ${ }^{6}$. These cases showing conus medullaris located at L3 or below, which are rare, are called as low-placed conus medullaris. Nevertheless, we did not see any cutaneous depression or hirsuteness that are characteristic of low-placed conus medullaris in the subjects, and thus we could hardly decide these cases low-placed conus medullaris. In the elderly people, lower end of conus medullaris situates relatively lower caused by senile degenerative change of intervertebral disks ${ }^{10)}$.

It was reported that morphological abnormalities such as marked superior extension of the sacral hiatus and elderly patients with low-placed caudal end of dura mater. In our present study, we found that the lower end of dura mater was located at or below S3 in $3.6 \%$ of cases. Typically, epidural puncture through the sacrum is frequently achieved by advancing the needle through the sacral hiatus and reducing the insertion angle of the needle on the cranial side in the midline position ${ }^{11)}$. Hence, on performing puncture of the sacral epidural space, there is a heightened risk of entry into the subarachnoid or subdural space when the needle is deeply inserted with a superior orientation. Given this danger, paying attention to the location of the lower ends of the subdural and the subarachnoid spaces is a key consideration on sacral epidural block or anesthesia. Furthermore, there are as yet no systematic reports on the correlation between stature, seated height, posture, and the location of Jacoby's line. In the present research, we were unable to investigate these factors. One of the reason is being impossible to measure the seated height of the cadavers due to postmortem rigidity-induced hardening.

In the present study, we could not clarify the positional changes of spinal cord and lower end of dura mater in accordance with vertebral motion. For solution of this issue, the necessity for a radiographic study is strongly suggested.

\section{Conclusions}

We observed the location of conus medullaris, the lower end of dura mater, and Jacoby's line in 60 cadavers. We found Jacoby's line passed through the level superior to L3 in $2(2.1 \%)$ of 48 subjects, Conus medullaris was inferior to L3 in $2(3.5 \%)$ of 57 subjects, and lower end of dura mater was located below the superior border of S3 in $1(3.6 \%)$ 28 subjects in which it could be accurately identified. On establishing Jacoby's line as usual, the site for subarachnoid puncture is settled in the L3-4 interspinous space, but when we consider that conus medullaris is located inferior to L 3 in $3.5 \%$ of cases, the possibility of injury to conus medullaris must be carefully considered. Accordingly, when attempting subarachnoid puncture in the elderly, it is important to avoid the higher lumbar levels as puncture sites whenever feasible.

\section{References}

1) Jacoby GW. Lumber puncture of the subarachnoid space. NY med J Dec 1895; 28:813-818. 
2) Jacoby GW. Lumber puncture of the subarachnoid space. NY med J Jan 1896; 4:6-12.

3) Kida S. An experience of Beer's spinal anaesthesia in Department of Surgery in Ogata Hospital. Tokyo Igakukai Zasshi 1907; 21:724-725. (in Japanese)

4) Matsuki A. Pioneers in Anaesthesiology. Tokyo, Kokuseidou, 1983; 267-280. (in Japanese)

5) Aono K. Spinal anaesthesia. Encyclopedia of Anaesthesiology (II). Tokyo, Kokuseidou, 1985; 825-841. (in Japanese)

6) Yokoyama K. Spinal Anesthesia. Tokyo, Shindan to Chiryosha 2000; 17-49. (in Japanese)
7) Moriyasu N. Modern Neurosurgery. Tokyo, Kanehara Shuppan, 1978; 134-135. (in Japanese)

8) Matsuo C, Goto M and Yokoyama K. Re-consideration of Jacoby's line. Abstract of 39th congress of Japanese society of Anaesthesiologists. Tokyo, Kokuseidou, 1992; S481. (in Japanese)

9) Render CE. The reproducibility of the iliac crest as a marker of lumber spine level. Anaesthesia 1996; 51:1070 1071.

10) Kikuchi S. Low Back Pain. Tokyo, Igaku Shoin, 2003; $27-$ 28. (in Japanese)

11) Kosaka Y. Clinical Epidural Anaesthesia. Tokyo, Kokuseidou, 1997; 28. (in Japanese) 